\title{
Simultaneously enhancing moisture and mechanical stability of flexible perovskite solar cells via a polyimide interfacial layer
}

\author{
Zhuoxi Li', Xiangyu Kong', Yue Jiang ${ }^{1,2}$, Xubing Lu', Xinseng Gao', Chaoliang Tan $^{3}$, Yiwang Chen ${ }^{4}$, Guofu \\ Zhou ${ }^{5}$, Jun-Ming Liu', Jinwei Gao' \\ IInstitute for Advanced Materials and Guangdong Provincial Key Laboratory of Optical Information Materials and Technology, \\ South China Academy of Advanced Optoelectronics, South China Normal University, Guangzhou 510006, Guangdong, China. \\ ${ }^{2}$ Key Lab of Functional Molecular Engineering of Guangdong Province, South China University of Technology, Guangzhou \\ 510640, Guangdong, China. \\ ${ }^{3}$ Department of Electrical Engineering, City University of Hong Kong, Kowloon, Hong Kong 999077, China. \\ ${ }^{4}$ Institute of Polymers and Energy Chemistry, College of Chemistry, Nanchang University, Nanchang 330031, Jiangxi, China. \\ ${ }^{5}$ Guangdong Provincial Key Laboratory of Optical Information Materials and Technology \& Institute of Electronic Paper Displays, \\ South China Academy of Advanced Optoelectronics, South China Normal University, Guangzhou 510006, Guangdong, China. \\ ${ }^{6}$ Laboratory of Solid-State Microstructures, Nanjing University, Nanjing 210093, Jiangsu, China.
}

Correspondence to: Prof. Jinwei Gao, Institute for Advanced Materials and Guangdong Provincial Key Laboratory of Optical Information Materials and Technology, South China Academy of Advanced Optoelectronics, South China Normal University, Guangzhou 510006, Guangdong, China. E-mail: gaojinwei@m.scnu.edu.cn; Dr. Yue Jiang, Institute for Advanced Materials and Guangdong Provincial Key Laboratory of Optical Information Materials and Technology, South China Academy of Advanced Optoelectronics, South China Normal University, Guangzhou 510006, Guangdong, China. E-mail: yuejiang@m.scnu.edu.cn

How to cite this article: Li Z, Kong X, Jiang Y, Lu X, Gao X, Tan C, Chen Y, Zhou G, Liu JM, Gao J. Simultaneously enhancing moisture and mechanical stability of flexible perovskite solar cells via a polyimide interfacial layer. Soft Sci 2021;1:4.

https://dx.doi.org/10.20517/ss.2021.06

Received: 5 Jul 2021 First Decision: 14 Jul 2021 Revised: 29 Jul 2021 Accepted: 30 Jul 2021 First online: 31 Jul 2021

Academic Editor: Zhifeng Ren Copy Editor: Xi-Jun Chen Production Editor: Xi-Jun Chen

\begin{abstract}
Perovskite solar cells (PSCs) have aroused tremendous attention due to the high power conversion efficiency (PCE) and flexibility of the organic-inorganic hybrid perovskite films. However, the commercialization of perovskite solar cells is still impeded due to the instability issue induced by moisture and mechanical stress. Herein, we introduce soluble hydrophobic polyimide (PI) as an interfacial layer on top of the perovskite film to block the infiltration of moisture into the perovskite film. The $\mathrm{MAPbl}_{3}$-based solar cell with the insertion of PI layer exhibited an impressive stability, maintaining $87 \%$ of the initial PCE even after exposing to $50 \%$ relative humidity for $550 \mathrm{~h}$ and presenting a decent PCE of $21.22 \%$ due to its ability to extract holes and reduce trap-assisted recombination. Moreover, the high tolerance of PI to the mechanical stress gives a more stable flexibility to the PSCs under
\end{abstract}


constant bending.

Keywords: Stability, hydrophobic, charge extraction, perovskite solar cells

\section{INTRODUCTION}

Organic-inorganic hybrid metal halide perovskite (OMHP) materials, as one of the most promising lightharvesting materials for photovoltaic devices, have been widely applied in perovskite solar cells (PSCs) by their virtues of outstanding optoelectronic properties and easy fabricating process ${ }^{[1-3]}$. Further, the flexibility of PSCs can help their integration with other power sources in wearable electronics ${ }^{[4]}$. To date, the certified power conversion efficiency (PCE) has reached $25.5 \%^{[5-7]}$, steadily approaching the performance of monocrystalline silicon solar cells. Nevertheless, the instabilities of OMHP caused by heat, oxygen, moisture, or mechanical stress tremendously hinder the commercialization of PSCs ${ }^{[8-10]}$.

Considering the ionic interaction in OMHP, water is the dominating factor during the degradation process at ambient atmosphere. As for methylammonium lead triiodide $\left(\mathrm{MAPbI}_{3}\right)$, its exposure to moisture would quickly and reversibly transform the original perovskite phase into intermediate monohydrates or even dihydrates byproducts, which would further irreversibly decompose into $\mathrm{CH}_{3} \mathrm{NH}_{3} \mathrm{I}$ and $\mathrm{PbI}_{2}$, as illustrated in Equations (1) and (2) ${ }^{[11]}$.

$$
\begin{aligned}
& 4\left(\mathrm{CH}_{3} \mathrm{NH}_{3}\right) \mathrm{PbI}_{3}+4 \mathrm{H}_{2} \mathrm{O} \rightleftharpoons 4\left[\mathrm{CH}_{3} \mathrm{NH}_{3} \mathrm{PbI}_{3} \cdot \mathrm{H}_{2} \mathrm{O}\right] \\
& \rightleftharpoons\left(\mathrm{CH}_{3} \mathrm{NH}_{3}\right) 4 \mathrm{PbI}_{6} \cdot 2 \mathrm{H}_{2} \mathrm{O}+3 \mathrm{PbI}_{2}+2 \mathrm{H}_{2} \mathrm{O} \\
&\left(\mathrm{CH}_{3} \mathrm{NH}_{3}\right)_{4} \mathrm{PbI}_{6} \cdot 2 \mathrm{H}_{2} \mathrm{O}(s) \stackrel{\mathrm{H}_{2} \mathrm{O}(\mathrm{l})}{\longrightarrow} 4 \mathrm{CH}_{3} \mathrm{NH}_{3} \mathrm{I}(q q)+\mathrm{PbI}_{2}(s)+2 \mathrm{H}_{2} \mathrm{O}(l)
\end{aligned}
$$

To prevent the perovskite from being destroyed by moisture, a possible solution is to utilize hydrophobic hole-transporting materials (HTMs). For example, Xie et al. ${ }^{[12]}$ introduced the hydrophobic and dopant-free Co-Ni bimetal-doped carbon aerogels simultaneously as HTM and electrode, remarkably improving the long-term stability of perovskite: 86\% of the initial efficiency was maintained even after $1296 \mathrm{~h}$ exposure in air. Similarly, Zheng et al. ${ }^{[13]}$ and Li et al. ${ }^{[14]}$ adopted the hydrophobic $2 \mathrm{D}$ perovskite materials as HTM and thus enhanced the moisture resistance of perovskite. Despite their excellent moisture stability, these stable devices always suffered from low efficiencies compared with the devices based on spiro-OMeTAD HTM. Instead, when a small amount of $2 \mathrm{D}$ perovskite is added into $3 \mathrm{D}$ perovskite, both moisture stability and photoelectric performance can be improved ${ }^{[15,16]}$.

Another important strategy is to apply effective water barrier polymer layers to encapsulate the perovskite film. Kim et al. ${ }^{[17]}$ demonstrated that hygroscopic polyethylene oxide can absorb water before the perovskite layer, preventing water from permeating into the perovskite film. On the other hand, a hydrophobic polymer layer can also protect perovskite from water penetration. Zhang's group deposited polystyrene on top of the perovskite layer, maintaining $73 \%$ of the initial PCE after $60 \mathrm{~h}$ in $80 \%$ humidity ${ }^{[18]}$. These approaches show the feasibility of employing a polymer as a functional layer to enhance the stability of perovskite films. Polyimide (PI) is a kind of multifunctional engineering materials with a high tolerance to temperature and mechanical stress. PI has been used as an additive to enhance the photovoltaic performance of PSCs ${ }^{[19]}$ and as foldable substrates in flexible devices ${ }^{[0,21]}$. Nevertheless, few studies apply PI as an interfacial layer. 
We introduced a soluble hydrophobic polymer, PI, by a spin-coating deposition method on top of perovskite films. By employing PI as the interfacial layer, a PCE of $21.22 \%$ was achieved based on $\mathrm{MAPbI}_{3}$, which could maintain $87 \%$ of the original PCE after more than $550 \mathrm{~h}$ aging in $50 \%$ relative humidity (RH) and $72 \%$ of the original PCE after 2500 cycles of bending. Further detailed studies showed that PI layer not only exhibits an excellent ability in extracting holes but also passivates the surface traps.

\section{EXPERIMENTAL}

\section{Material and solvent}

Methylammonium iodide (MAI, 99.5\%) was purchased from Xi'an Polymer Light Technology Corp. Lead iodide $\left(\mathrm{PbI}_{2}, 99.999 \%\right)$ and 2,2',7,7'-tetrakis(N,N-di-p-methoxyphenylamine)-9,9-spirobifluorene (spiroOMeTAD, 99.8\%) were purchased from Yingkou Libra New Energy Technology Co., Ltd. The aqueous dispersion of PEDOT:PSS (AI 4083) was obtained from Shanghai MaterWin New Materials Co., Ltd. Bis(trifluoromethanesulfonyl)imide (Li-TFSI, 99.5\%), tert-butylpyridine (t-BP, 98\%), and Tin(II) chloride dihydrate $\left(\mathrm{SnCl}_{2} \cdot 2 \mathrm{H}_{2} \mathrm{O}, 98 \%\right)$ were purchased from Sigma-Aldrich. A soluble polyimide (PI, $5 \mathrm{wt} \%$ in NMethylpyrrolidone) was obtained from ShenZhen Dalton Electronic Material Co., Ltd. As for solvent, dimethyl formamide (DMF, 99.8\%), dimethyl sulfoxide (DMSO, 99.9\%), chlorobenzene (CB, 99.8\%), acetonitrile (99.8\%), and butanol (99.8\%) were purchased from Sigma-Aldrich and used without further purification. FTO glasses (FTO, $7 \Omega$ per square) were purchased from Advanced Election Technology Co., Ltd.

\section{Solution preparation}

The $\mathrm{SnO}_{2}$ nanocrystalline (NC) solution was prepared by our previous method ${ }^{[22]}$. Typically, the $\mathrm{SnCl}_{2} \cdot 2 \mathrm{H}_{2} \mathrm{O}$ solution $(0.1 \mathrm{M})$ was prepared by dissolving $\mathrm{SnCl}_{2} \cdot 2 \mathrm{H}_{2} \mathrm{O}$ separately in $20 \mathrm{~mL}$ butanol containing $2 \mathrm{~mL}$ water in a flask. Then, the solution was separately stirred at room temperature for $10 \mathrm{~min}$. To synthesize colloidal $\mathrm{SnO}_{2} \mathrm{NC}$, the $\mathrm{SnCl}_{2} \cdot 2 \mathrm{H}_{2} \mathrm{O}$ solution was refluxed at $110^{\circ} \mathrm{C}$ for $2-4 \mathrm{~h}$ with an open refluxing apparatus.

The perovskite precursor was prepared by dissolving $922 \mathrm{mg} \mathrm{PbI}_{2}$ and $318 \mathrm{mg}$ MAI into $1.6 \mathrm{~mL}$ mixed solvent of DMF and DMSO $(7: 3 \mathrm{v} / \mathrm{v})$. Spiro-OMeTAD solution was prepared by dissolving $72 \mathrm{mg}$ spiroOMeTAD into $1 \mathrm{~mL} \mathrm{CB}$, with the dopant of $17.5 \mu \mathrm{L}$ Li-TFSI solution (520 mg in $1 \mathrm{~mL}$ acetonitrile) and $28.8 \mu \mathrm{L}$ t-BP.

\section{Perovskite solar cells fabrication}

The FTO substrates were sequentially ultrasonically cleaned with detergent, deionized water, and isopropanol for $15 \mathrm{~min}$, respectively. Before spin-coating $\mathrm{SnO}_{2}$ ETLs, the FTO substrates were further cleaned by UV-ozone for $15 \mathrm{~min}$. Then, the $\mathrm{SnO}_{2} \mathrm{NC}$ solution was dropped on the FTO substrates and spin-coated at $500 \mathrm{rpm}$ for $3 \mathrm{~s}$ and $3000 \mathrm{rpm}$ for $30 \mathrm{~s}$, followed by thermal annealing at $150{ }^{\circ} \mathrm{C}$ for $1 \mathrm{~h}$. After the substrates cooled down, a 15 min UV-ozone treatment was operated again. Then, all substrates were transfer into a glovebox filled with $\mathrm{N}_{2}$ atmosphere to complete the subsequent process. The perovskite precursor solution was spin-coated on $\mathrm{SnO}_{2}$ layer at $500 \mathrm{rpm}$ for $3 \mathrm{~s}$ and $4000 \mathrm{rpm}$ for $30 \mathrm{~s}$. Then, $400 \mu \mathrm{L} \mathrm{CB}$ as antisolvent were dripped onto the perovskite film $21 \mathrm{~s}$ prior to the end of the second spinning program. Afterward, the perovskite film was annealed on hotplate at $100{ }^{\circ} \mathrm{C}$ for $10 \mathrm{~min}$. PI solution $(0.5,1.0,1.5$, and $2.0 \mathrm{wt} \%$ in $\mathrm{CB}$ ) was spin-coated at $500 \mathrm{rpm}$ for $3 \mathrm{~s}$ and $4000 \mathrm{rpm}$ for $30 \mathrm{~s}$, followed by 20 min annealing treatment at $100{ }^{\circ} \mathrm{C}$. When it cooled down, spiro-OMeTAD solution was spin-coated upon the perovskite film at $500 \mathrm{rpm}$ for $3 \mathrm{~s}$ and $3000 \mathrm{rpm}$ for $30 \mathrm{~s}$. Finally, the Ag electrode was thermally evaporated on top of the device under high vacuum condition $\left(<5 \times 10^{-4} \mathrm{~Pa}\right)$. The active area of all devices was $0.07 \mathrm{~cm}^{2}$ defined by a metal mask. 


\section{Characterization}

The surface and cross-section morphologies of perovskite and PSCs devices were investigated by FE-SEM (ZEISS Ultra-55). The ultraviolet and visible spectrophotometry (UV-vis) absorption spectra of perovskite film were measured by SHIMADZU UV-2700 in the wavelength range of 450-850 $\mathrm{nm}$. The surface roughness of the perovskite film was obtained from atomic force microscope (AFM) (Asylum Research, Cypher). The water contact angle of the perovskite surface was measured by contact angle tester (Dataphysics OCA20, German). Ultraviolet photoelectron spectroscopy was performed on SHIMADZU AXIS SUPRA. The Fourier transform infrared (FTIR) spectrum was conducted on Bruker Vertex 70. The current-voltage $(\mathrm{J}-\mathrm{V})$ curves of the non-encapsulated solar cells were measured by Keithley 2400 by forward scan $(1.2$ to $-0.2 \mathrm{~V}$ ) with a scanning rate of $50 \mathrm{mV} / \mathrm{s}$ (voltage step of $10 \mathrm{mV}$ and delay time of $50 \mathrm{~ms}$ ) in a glovebox under AM $1.5 \mathrm{G}$ illuminations $\left(100 \mathrm{~mW} \cdot \mathrm{cm}^{-2}\right)$ from a solar simulator (Newport, 91160), which was calibrated using a standard silicon solar cell device by the NREL. Steady-state photoluminescence (PL) spectra were measured under an excitation wavelength of $460 \mathrm{~nm}$ by using Lengguang F97pro Fluorescence Spectrophotometer. Time-resolved photoluminescence (TRPL) spectra were recorded by a spectrofluorometer (Edinburgh FLS9800) under a $450 \mathrm{~nm}$ excitation. Electrochemical impedance spectroscopy (EIS) was studied on an electrochemical workstation (CHI660) from $1 \mathrm{MHz}$ to $1 \mathrm{~Hz}$, biased at $0.2 \mathrm{~V}$ in the dark. X-ray diffraction (XRD) (PANalytical X'Pert PRO) was performed with Cu Ka radiation under operating conditions of $40 \mathrm{kV}$ and $40 \mathrm{~mA}$. The space charge limited current (SCLC) characteristic of the devices was measured using a Keithley 2400 in the dark. According to SCLC theory, the defect (trap) density $\left(n_{\text {trap }}\right)$ can be estimated as follows: $n_{\text {trap }}=\left(2 \varepsilon \varepsilon_{0} V_{\text {TFL }}\right) /\left(e L^{2}\right)$, where $L, \varepsilon, \varepsilon_{0}$, and $e$ are the thickness of the perovskite film (ca. $530 \mathrm{~nm}$ ), dielectric constant of the material, permittivity of vacuum, and electronic charge, respectively. The external quantum efficiency (EQE) measurements of devices were carried out using QE-C system (Taiwan, Enlitech) in a wavelength range of 300-900 $\mathrm{nm}$.

\section{RESULTS AND DISCUSSION}

The concentration of the PI was optimized by characterizing the morphology of PI films on top of the perovskite with the top-view scanning electron microscopy (SEM), as shown in Figure 1A-E. The pristine perovskite film [Figure 1A] shows a compact surface with grain sizes ranging from 200 to $600 \mathrm{~nm}$. After combining the PI layer [Figure 1B-E], the morphologies of perovskite films have no apparent changes compared with that of pristine film. Specially, PI at lower concentration ( $0.5 \mathrm{wt} \%)$ gives a relatively flat and continuous morphology, which was also confirmed by the AFM, as illustrated in Supplementary Figure 1. The water contact angles were largely changed after the inset of the PI layer on top of the perovskite films, as shown in Figure 1F-J. Due to the high hydrophobicity of PI, the contact angle of perovskite was sharply increased from $56.9^{\circ}$ (pristine) to $95.3^{\circ}$ (PI layer at the concentration of $2 \mathrm{wt} \%$ ) upon the insertion of PI interfaces, providing the possibility of efficient inhibiting of moisture degradation ${ }^{[23]}$. By measuring the ultraviolet photoelectron spectroscopy curves of the perovskite films with and without PI, the energy level diagram of the PSC could be drawn [Supplementary Figure 2]. Due to the thin thickness of PI $(\sim 10 \mathrm{~nm}$, as shown in Supplementary Figure 3), holes from the value band maximal of perovskite can tunnel to spiro$\mathrm{OMeTAD}^{[24,25]}$. The FTIR spectrum of pure PI is presented in Supplementary Figure 4, showing the characteristic band peaks of the imide carbonyl were $1779 \mathrm{~cm}^{-1}$ (asymmetric $\mathrm{C}=\mathrm{O}$, stretching), $1721 \mathrm{~cm}^{-1}$ (symmetric $\mathrm{C}=\mathrm{O}$, stretching), and $1362 \mathrm{~cm}^{-1}(\mathrm{C}-\mathrm{N} \text {, stretching })^{[26,27]}$.

Planar PSCs with configuration of $\mathrm{FTO} / \mathrm{SnO}_{2} / \mathrm{MAPbI}_{3} / \mathrm{PI} /$ spiro-OMeTAD/Ag were fabricated, with the thickness of $\mathrm{SnO}_{2}, \mathrm{MAPbI}_{3}$, spiro-OMeTAD, and Ag being about 30, 300, 140, and $80 \mathrm{~nm}$, respectively, as shown in the cross-sectional SEM image [Supplementary Figure 5]. These PSCs were then measured under an irradiation intensity of $100 \mathrm{~mW} / \mathrm{cm}^{2}$ (AM $1.5 \mathrm{G}$ solar spectrum), and the current density-voltage curves $(J-V)$ along with the related parameters are illustrated in Figure 2A. As the concentration of PI increased from $0 \mathrm{wt} \%$ to $2 \mathrm{wt} \%$, the corresponding PCE of the devices was first enhanced from $20.72 \%$ ( $V_{\mathrm{OC}}$ of $1.17 \mathrm{~V}$, 


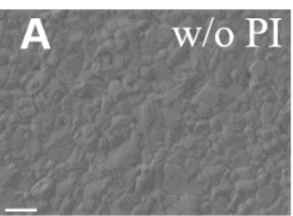

F

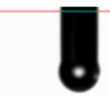

CA left: $56.9^{\circ}$

CA right: $57.6^{\circ}$

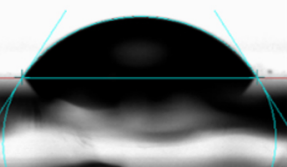

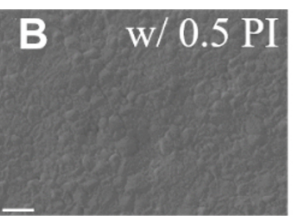

G

CA left: $69.8^{\circ}$

CA right: 69.3

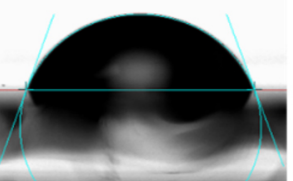

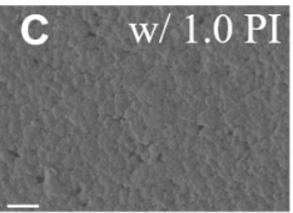

H

CA left: $72.9^{\circ}$

CA right: 72.3

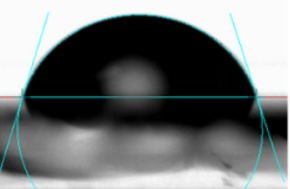

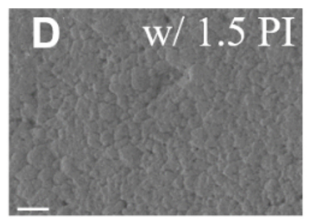

I

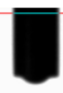

CA left: $96.5^{\circ}$

CA right: $96.6^{\circ}$

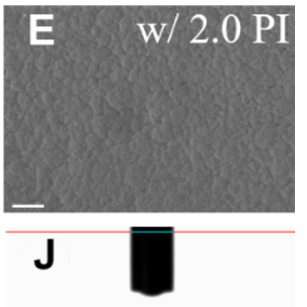

CA left: $95.3^{\circ}$

CA right: $95.9^{\circ}$
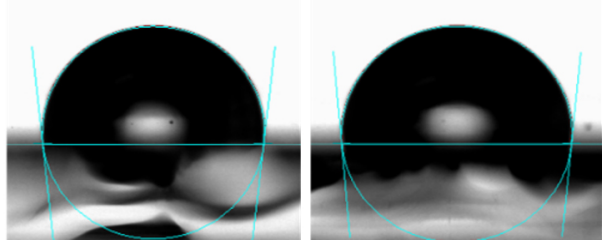

Figure 1. Top-view SEM images of pristine perovskite films (A). Top-view SEM images of perovskite films modified by PI solution with different concentrations: (B) $0.5 \mathrm{wt} \%$; (C) $1.0 \mathrm{wt} \%$; (D) $1.5 \mathrm{wt} \%$; and (E) $2.0 \mathrm{wt} \%$. The corresponding water contact angles based on the above perovskite films: (F) for (A); (G) for (B); (H) for (C); (I) for (D); and (J) for (E). SEM: Scanning electron microscopy. Pl: polyimide.
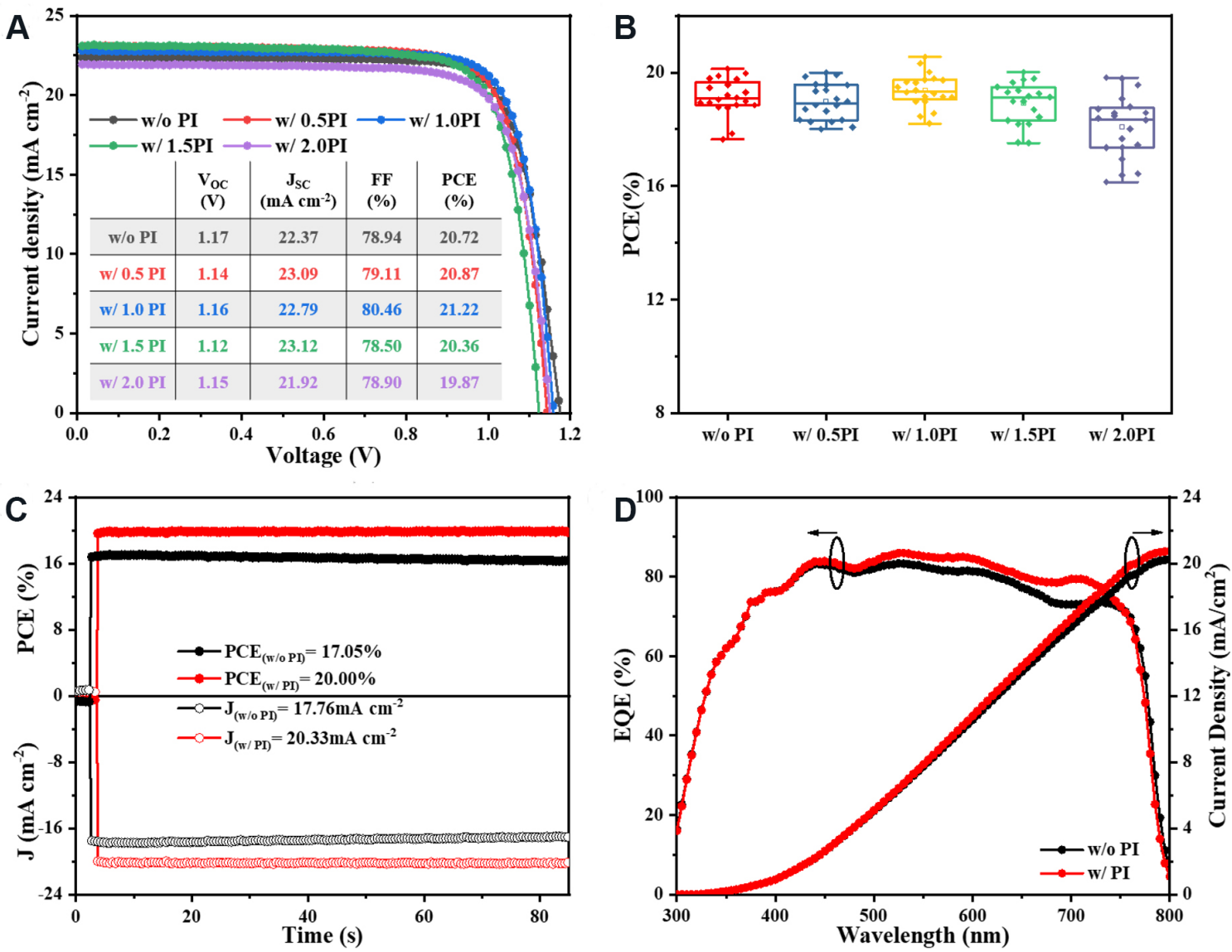

Figure 2. (A) J-V curves and (B) the PCE statistical distributions of 20 devices with different concentrations of PI. (C) The steady-state photocurrent measurements at maximum power point. (D) EQE spectra for the devices with $(w /)$ ) and without (w/o) PI. PCE: High power conversion efficiency; EQE: external quantum efficiency; PI: polyimide. 
$J_{\mathrm{SC}}$ of $22.37 \mathrm{~mA} / \mathrm{cm}^{2}$, and $\mathrm{FF}$ of $78.94 \%$ ) at $0 \mathrm{wt} \%$ to $21.22 \%\left(V_{\mathrm{OC}}\right.$ of $1.16 \mathrm{~V}, J_{\mathrm{SC}}$ of $22.79 \mathrm{~mA} / \mathrm{cm}^{2}$, and FF of $80.46 \%$ ) at $1 \mathrm{wt} \%$, while the PCE decreased to $19.87 \%$ ( $V_{\mathrm{OC}}$ of $1.15 \mathrm{~V}, J_{\mathrm{SC}}$ of $21.92 \mathrm{~mA} / \mathrm{cm}^{2}$, and $\mathrm{FF}$ of $78.90 \%$ ) when 2 wt\% of PI was used, which is considered to have arisen by the charge blocking effect of thick PI films ${ }^{[28]}$.

The statistical PCE distribution of 20 independent devices fabricated from PI solution shown in Figure $2 \mathrm{~B}$ suggests the same trend as the $J-V$ curves. Besides, the devices based on $1.0 \mathrm{wt} \%$ PI exhibit the smallest variation and the best reproducibility. Therefore, it was chosen to compare with the PI-free device to the device with PI in the subsequent characterization, denoted as w/o PI and w/ PI, respectively.

To further confirm the values obtained from $J$ - $V$ curves, steady-state PCE was recorded over $85 \mathrm{~s}$ under the maximum power point voltage ( 0.98 and $0.96 \mathrm{~V}$ for devices w/ PI and w/o PI), as shown in Figure $2 \mathrm{C}$, giving a PCE of $20.00 \%$ with the stabilized $J_{\mathrm{SC}}$ of $20.33 \mathrm{~mA} / \mathrm{cm}^{2}$ and a PCE of $17.05 \%$ with the stabilized $J_{\mathrm{SC}}$ of $17.76 \mathrm{~mA} / \mathrm{cm}^{2}$. Furthermore, the integrated $J_{\mathrm{SC}}$ from EQE curves of devices w/ PI and w/o PI in Figure 2D are 20.76 and $20.29 \mathrm{~mA} / \mathrm{cm}^{2}$, respectively, which agrees well with the $J-V$ curves.

The dynamics of photo-excited carriers was investigated to understand the origin of the PCE enhancement after inserting this high-dielectric PI by PL and TRPL measurement, as shown in Figure 3A. A strong luminescence peak at $780 \mathrm{~nm}$ was observed, which was significantly decreased upon the insertion of PI interface, demonstrating the improved charge transfer efficiency. In addition, the PL lifetime was obtained by fitting the TRPL [Figure 3B] spectra with a two-exponential decay model: $I(t)=A_{1} \exp \left(-t / \tau_{1}\right)+A_{2} \exp \left(-t / \tau_{2}\right)$ $+I_{0}$. The fast decay lifetimes $\left(\tau_{1}\right)$ represent the nonradiative recombination induced by the trapping process when the charge goes through the perovskite surface, and the slow decay lifetimes $\left(\tau_{2}\right)$ are related to the radiative recombination process occurring in the bulk perovskite ${ }^{[29-31]}$. The fitted parameters are summarized in Supplementary Table 1. In this case, the average charge lifetime $\left(\tau_{\text {avg }}\right)$ was estimated to be 220.9 and $32.1 \mathrm{~ns}$ for devices w/o PI and w/ PI, respectively, apparently confirming that the PI layer is greatly beneficial for the hole-extraction process ${ }^{[32]}$ and contributes to the increase of the FF.

Subsequently, to assess the hole-trap density at the perovskite/spiro-OMeTAD interfaces modified by PI layer, the SCLC method was conducted based on the hole-only devices, FTO/PEDOT:PSS/perovskite/with or without PI layer/spiro-OMeTAD/Ag. As shown in Figure $3 \mathrm{C}$, the ohmic conducting region at lower voltage and trap-filled limited (TFL) region at higher voltage intersect at one point, where its corresponding voltage is the trap-filled limit voltage $\left(V_{\mathrm{TFL}}\right)^{[33]}$, which is reported positively correlated with the trap density $\left(n_{\text {traps }}\right)^{[34]}$. In Figure 3C, the $V_{\text {TFL }}$ of devices w/o PI and w/ PI was measured to be 0.84 and $0.77 \mathrm{~V}$, corresponding to a $n_{\text {traps }}$ of $9.31 \times 10^{15}$ and $8.22 \times 10^{15} \mathrm{~cm}^{-3}$, respectively. Thus, we could conclude that inserting PI is an effective strategy for the suppression of traps, further favoring the efficient charge transfer at the perovskite/spiro-OMeTAD interfaces.

Moreover, from the Nyquist plots of PSCs in Figure 3D, obtained by EIS measurement in the dark, the semi-circle of the device w/ PI exhibits a smaller radius in the high frequency region, which demonstrates a lower charge transfer resistance $\left(R_{\mathrm{ct}}\right)$. Simultaneously, the radius of its semi-circle in the low frequency region associated with the recombination resistance $\left(R_{\mathrm{rec}}\right)^{[35]}$ is bigger than that of device w/o PI, consistent with the conclusion that the trap was mitigated by the PI interfaces.

Finally, the influence of PI interfaces on the moisture and mechanical stability is shown in Figure 4. Firstly, $\mathrm{MAPbI}_{3}$ was deposited on the FTO substrate, followed by the deposition of the PI polymer. After a certain exposure time under high $\mathrm{RH}$ of $75 \%$ at $25{ }^{\circ} \mathrm{C}$, the degree of degradation could be observed from the UV-vis 
A
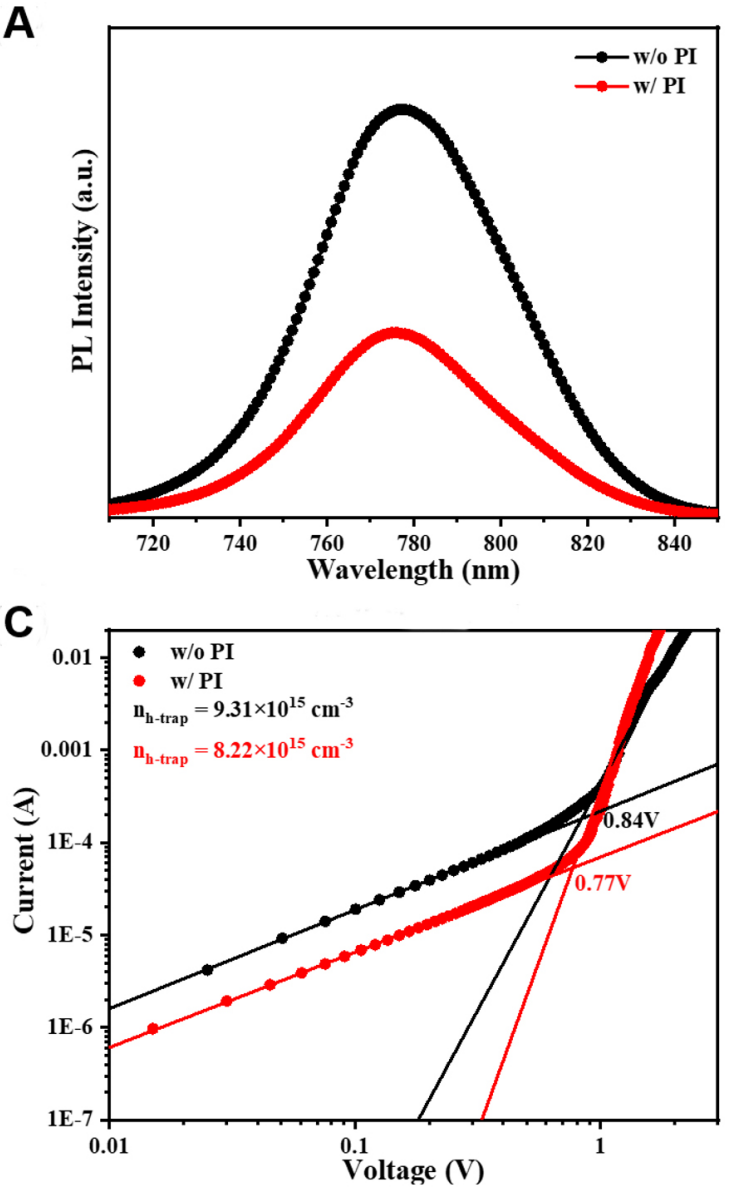
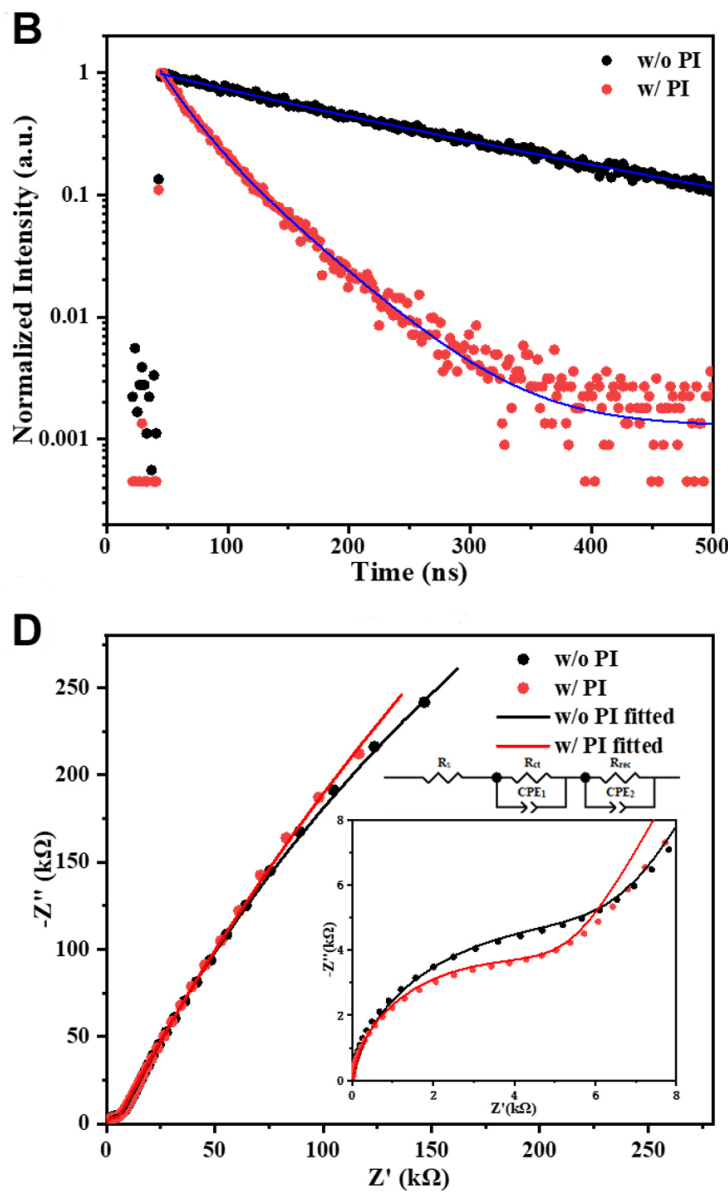

Figure 3. (A) PL spectra and (B) TRPL decay curves for the devices with (w/) and without (w/o) PI. (C) The dark current-voltage curves for hole-only devices with (w/) and without (w/o) PI. (D) Nyquist plots of the devices with (w/) and without (w/o) Pl, measured in the dark. TRPL: Time-resolved photoluminescence; PI: polyimide; PL: photoluminescence.

absorption curve, as shown in Figure 4A. Both films showed a similar high absorbance on the first day, indicating that the introduction of PI layer has little effect on light absorption. After six days of exposure, the absorbance of the perovskite film w/o PI dropped sharply, while the PI-modified film gave little change. Simultaneously, XRD measurement was conducted to trace the phase transition of perovskite films. Figure $4 \mathrm{~B}$ shows that the fresh w/o PI and w/ PI films presented main diffraction peaks of perovskite at $14.12^{\circ}$. However, in the spectra of aged perovskite film w/o PI (75\% RH for four days), obvious peaks at $12.57^{\circ}, 10.17^{\circ}$, and $8.40^{\circ}$, ascribed to $\mathrm{PbI}_{2}$ and monohydrate perovskite, were detected ${ }^{[1,36]}$, forming a sharp contrast with the film w/ PI. Thereby, it is considered that the insertion of the PI layer indeed protects the perovskite film from fast moisture intrusion in high-humidity environments.

The long-term stability of PSCs in ambient condition was evaluated by monitoring the PCE of the devices without any encapsulation under $50 \% \mathrm{RH}$ at $25{ }^{\circ} \mathrm{C}$. As shown in Figure $4 \mathrm{C}$, with PI treatment, the devices maintained $87 \%$ of their original PCE after aging over $550 \mathrm{~h}$, whereas the PCE of the pristine perovskite films dropped to $58 \%$ of initial PCE. The insert figures show the optical photos of perovskite films stored in the same condition, where transparent hydrate can be found in the PI-free film after aging. 

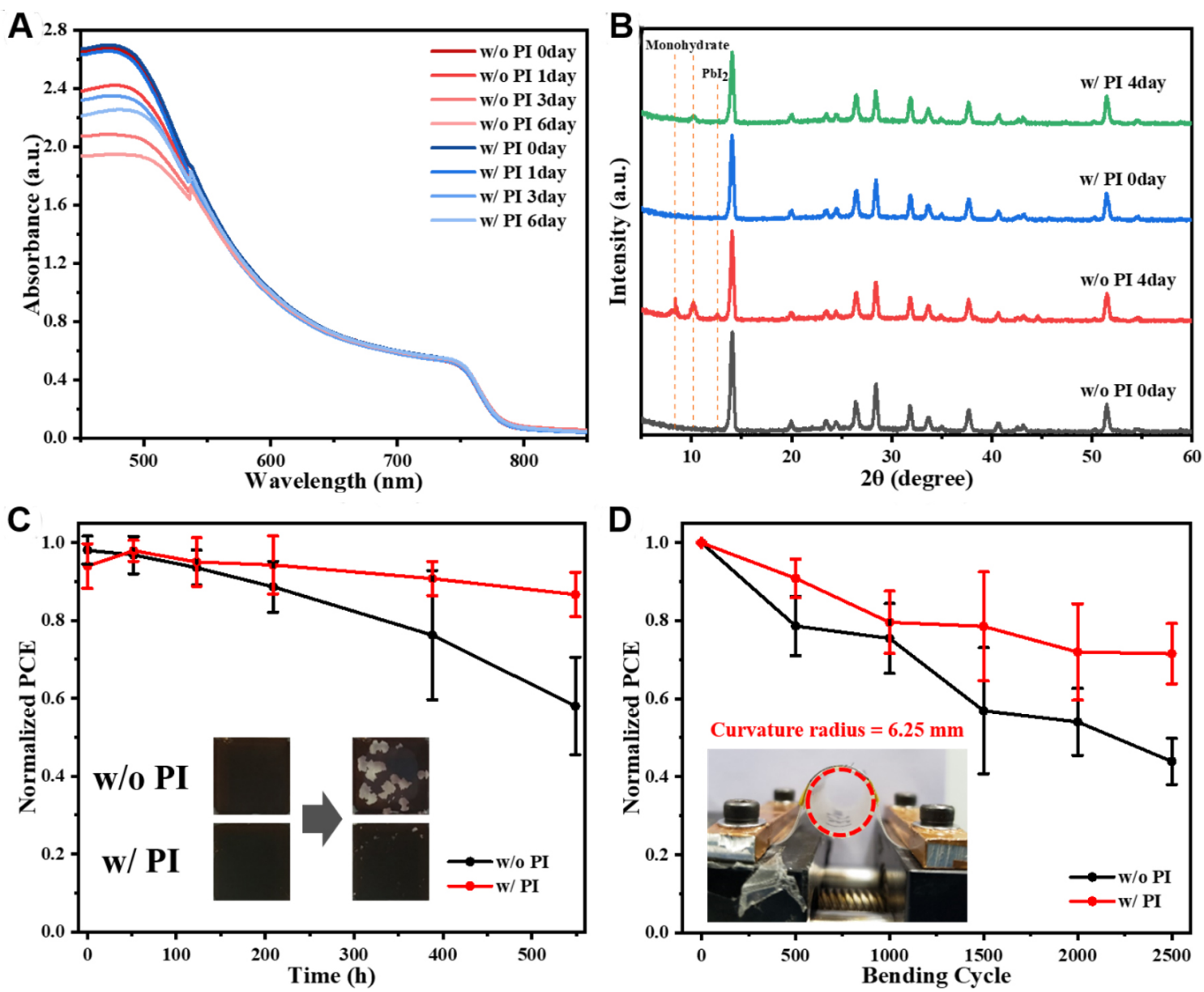

Figure 4. (A) Variations of the UV-vis absorption and (B) XRD measurement of perovskite without and with $\mathrm{PI}$ under an $\mathrm{RH}$ of $75 \%$ at $25^{\circ} \mathrm{C}$ in dark conditions with respect to the exposure duration. (C) Long-term stability of the six corresponding PSCs under an RH of $50 \%$ at $25{ }^{\circ} \mathrm{C}$ in dark conditions. The inserts are optical photographs. (D) Normalized average PCE of 10 PSCs as a function of stretching cycles under a curvature radius of $6.25 \mathrm{~mm}$. The inset shows the photograph of the flexible device under curvature. XRD: Xray diffraction $\mathrm{RH}$ : relative humidity; PSCs: perovskite solar cells; PI: polyimide.

Aiming to validate the mechanical stability of the devices, the flexible PSCs were fabricated based on polyethylene naphthalate (PEN)/ITO substrate with or without PI, which showed a PCE of $17.52 \%$ and $16.90 \%$, respectively [Supplementary Figure 6]. Figure 4D shows the normalized average PCE versus bending cycle under a curvature radius of $6.25 \mathrm{~mm}$. The PI-modified devices exhibit remarkable stretching tolerance, maintaining $72 \%$ of the original PCE after 2500 cycles, while the PSCs without PI only gave $44 \%$ of the pristine PCE, possibly due to the formation of crack on the perovskite film ${ }^{[37]}$. Supplementary Table 2 shows a comparison of the moisture stability and mechanical stability of PSCs assisted by polymers, where our work shows outstanding moisture and mechanical stability. Moreover, our PI presents a simultaneous enhancement of both aspects. Thus, it is concluded that the high tolerance of mechanical stress of PI on top of perovskite could take part in the applied mechanical extrusion, giving rise to the mitigated influence of external mechanical stress.

\section{CONCLUSIONS}

In summary, we introduced a soluble PI as an interfacial layer to enhance the moisture and mechanical resistance. Further investigations manifested that the PI layer not only extracted holes but also reduced trapassisted recombination at the interface, achieving a PCE of $21.22 \%$ in $\mathrm{MAPbI}_{3}$-based PSCs. Most 
importantly, at 50\% RH, the devices with PI layer maintained $87 \%$ of the initial PCE after aging over $550 \mathrm{~h}$, far superior to the pristine solar cells. Moreover, PI insertion enhanced the device tolerance against the external mechanical stress, providing an effective strategy to improve the stability of flexible PSCs.

\section{DECLARATIONS}

\section{Acknowledgments}

The authors gratefully acknowledge the funding support by NSFC Funds (U1801256, 51803064), Science and Technology Programs of Guangzhou (2019050001, 202002030130), International Cooperation Projects of Guangdong Province (2020A0505100054), Guangdong Provincial Key Laboratory of Optical Information Materials and Technology (2017B030301007), and Collaborative Research Fund (CRF C7018-20GF) of Hong Kong Research Grants Council. We also thank the support from the Guangdong Provincial Engineering Technology Research Center for Transparent Conductive Materials, and the Open Fund of the Key Laboratory of Functional Molecular Engineering of Guangdong Province (2018kfxx, South China University of Technology).

\section{Authors' contributions}

Contributions to conception and design of the study: Li Z, Jiang Y, Gao J

Data analysis and interpretation and data acquisition: Li Z, Kong X, Jiang Y, Gao J, Chen Y, Tan C

Administrative, technical, and material support: Gao J, Gao X, Lu X, Liu JM, Zhou G

Manuscript writing: Li Z, Jiang Y, Gao J

\section{Availability of data and materials}

Not applicable.

\section{Financial support and sponsorship}

National Natural Science Foundation of China.

\section{Conflicts of interest}

All authors declared that there are no conflicts of interest.

\section{Ethical approval and consent to participate}

Not applicable.

\section{Consent for publication}

Not applicable.

\section{Copyright}

(c) The Author(s) 2021.

\section{REFERENCES}

1. Zhang F, Zhu K. Additive engineering for efficient and stable perovskite solar cells. Adv Energy Mater 2020;10:1902579. DOI

2. Chen J, Park N. Materials and methods for interface engineering toward stable and efficient perovskite solar cells. ACS Energy Lett 2020;5:2742-86. DOI

3. Ran C, Xu J, Gao W, Huang C, Dou S. Defects in metal triiodide perovskite materials towards high-performance solar cells: origin, impact, characterization, and engineering. Chem Soc Rev 2018;47:4581-610. DOI PubMed

4. Zhao J, Xu Z, Zhou Z, et al. A safe flexible self-powered wristband system by integrating defective $\mathrm{MnO}_{2-\mathrm{x}}$ nanosheet-based Zinc-Ion batteries with perovskite solar cells. ACS Nano 2021;15:10597-608. DOI PubMed

5. National Renewable Energy Laboratory USA, Best research-cell efficiencies. Available from: https://www.nrel.gov/pv/cellefficiency.html. [Last accessed on 9 Aug 2021].

6. Jiang Q, Zhao Y, Zhang X, et al. Surface passivation of perovskite film for efficient solar cells. Nat Photonics 2019;13:460-6. DOI PubMed

7. Yoo JJ, Seo G, Chua MR, et al. Efficient perovskite solar cells via improved carrier management. Nature 2021;590:587-93. DOI 
PubMed

8. Wang R, Mujahid M, Duan Y, Wang Z, Xue J, Yang Y. A review of perovskites solar cell stability. Adv Funct Mater 2019;29:1808843. DOI

9. Niu T, Lu J, Munir R, et al. Stable high-performance perovskite solar cells via grain boundary passivation. Adv Mater 2018;30:e1706576. DOI PubMed

10. Choi K, Lee J, Kim HI, et al. Thermally stable, planar hybrid perovskite solar cells with high efficiency. Energy Environ Sci 2018;11:3238-47. DOI

11. Leguy AMA, Hu Y, Campoy-quiles M, et al. Reversible hydration of $\mathrm{CH}_{3} \mathrm{NH}_{3} \mathrm{PbI}_{3}$ in films, single crystals, and solar cells. Chem Mater 2015;27:3397-407. DOI

12. Xie Y, Cheng J, Liu H, et al. Co-Ni alloy@carbon aerogels for improving the efficiency and air stability of perovskite solar cells and its hysteresis mechanism. Carbon 2019;154:322-9. DOI

13. Zheng H, Liu G, Zhu L, et al. The effect of hydrophobicity of ammonium salts on stability of quasi-2D perovskite materials in moist condition. Adv Energy Mater 2018;8:1800051. DOI

14. Li F, Zhang J, Jo S, et al. Vertical orientated Dion-Jacobson quasi-2D perovskite film with improved photovoltaic performance and stability. Small Methods 2020;4:1900831. DOI

15. Wang Z, Lin Q, Chmiel FP, Sakai N, Herz LM, Snaith HJ. Efficient ambient-air-stable solar cells with 2D-3D heterostructured butylammonium-caesium-formamidinium lead halide perovskites. Nat Energy 2017;2:17135. DOI

16. Zhang T, Dar MI, Li G, et al. Bication lead iodide 2D perovskite component to stabilize inorganic $\alpha-\mathrm{CsPb}_{3}$ perovskite phase for highefficiency solar cells. Sci Adv 2017;3:e1700841. DOI PubMed PMC

17. Kim M, Motti SG, Sorrentino R, Petrozza A. Enhanced solar cell stability by hygroscopic polymer passivation of metal halide perovskite thin film. Energy Environ Sci 2018;11:2609-19. DOI

18. Zhang H, Shi J, Zhu L, et al. Polystyrene stabilized perovskite component, grain and microstructure for improved efficiency and stability of planar solar cells. Nano Energy 2018;43:383-92. DOI

19. Yu Y, Tseng C, Chien W, Hsu H, Chen C. Photovoltaic performance enhancement of perovskite solar cells using polyimide and polyamic acid as additives. $J$ Phys Chem C 2019;123:23826-33. DOI

20. Jeong G, Koo D, Seo J, et al. Suppressed interdiffusion and degradation in flexible and transparent metal electrode-based perovskite solar cells with a graphene interlayer. Nano Lett 2020;20:3718-27. DOI PubMed

21. Yoon J, Kim U, Yoo Y, et al. Foldable perovskite solar cells using carbon nanotube-embedded ultrathin polyimide conductor. $A d v S c i$ (Weinh) 2021;8:2004092. DOI PubMed PMC

22. Chen C, Jiang Y, Guo J, et al. Solvent-assisted low-temperature crystallization of $\mathrm{SnO}_{2}$ electron-transfer layer for high-efficiency planar perovskite solar cells. Adv Funct Mater 2019;29:1900557. DOI

23. Zhang S, Liu Z, Zhang W, et al. Barrier designs in perovskite solar cells for long-term stability. Adv Energy Mater 2020;10:2001610. DOI

24. Wang Q, Dong Q, Li T, Gruverman A, Huang J. Thin insulating tunneling contacts for efficient and water-resistant perovskite solar cells. Adv Mater 2016;28:6734-9. DOI PubMed

25. Wen X, Wu J, Ye M, Gao D, Lin C. Interface engineering via an insulating polymer for highly efficient and environmentally stable perovskite solar cells. Chem Commun (Camb) 2016;52:11355-8. DOI PubMed

26. Yang Z, Ma P, Li F, Guo H, Kang C, Gao L. Ultrahigh thermal-stability polyimides with low CTE and required flexibility by formation of hydrogen bonds between poly(amic acid)s. European Polymer Journal 2021;148:110369. DOI

27. Zhang J, Jiang P, Wang Y, Liu X, Ma J, Tu G. In situ synthesis of ultrastable CsPbBr ${ }_{3}$ perovskite nanocrystals coated with polyimide in a CSTR system. ACS Appl Mater Interfaces 2020;12:3080-5. DOI PubMed

28. Chen N, Yi X, Zhuang J, et al. An efficient trap passivator for perovskite solar cells: poly(propylene glycol) bis(2-aminopropyl ether). Nanomicro Lett 2020;12:177. DOI PubMed PMC

29. Huang Z, Hu X, Liu C, et al. Water-resistant and flexible perovskite solar cells via a glued interfacial layer. Adv Funct Mater 2019;29:1902629. DOI

30. Cai Y, Cui J, Chen M, et al. Multifunctional enhancement for highly stable and efficient perovskite solar cells. Adv Funct Mater 2021;31:2005776. DOI

31. Liu K, Liang Q, Qin M, et al. Zwitterionic-surfactant-assisted room-temperature coating of efficient perovskite solar cells. Joule 2020;4:2404-25. DOI

32. Chen C, Li F, Zhu L, et al. Efficient and stable perovskite solar cells thanks to dual functions of oleyl amine-coated $\mathrm{PbSO} 4(\mathrm{PbO}) 4$ quantum dots: Defect passivation and moisture/oxygen blocking. Nano Energy 2020;68:104313. DOI

33. Duijnstee EA, Ball JM, Le Corre VM, Koster LJA, Snaith HJ, Lim J. Toward understanding space-charge limited current measurements on metal halide perovskites. ACS Energy Lett 2020;5:376-84. DOI

34. Liu Z, Cao F, Wang M, Wang M, Li L. Observing defect passivation of the grain boundary with 2-aminoterephthalic acid for efficient and stable perovskite solar cells. Angew Chem Int Ed Engl 2020;59:4161-7. DOI PubMed

35. Zhao Y, Zhu P, Huang S, et al. Molecular interaction regulates the performance and longevity of defect passivation for metal halide perovskite solar cells. J Am Chem Soc 2020;142:20071-9. DOI PubMed

36. Yun S, Ma S, Kwon H, et al. Amino acid salt-driven planar hybrid perovskite solar cells with enhanced humidity stability. Nano Energy 2019;59:481-91. DOI

37. Duan X, Li X, Tan L, et al. Controlling crystal growth via an autonomously longitudinal scaffold for planar perovskite solar cells. $A d v$ Mater 2020;32:e2000617. DOI PubMed 